\title{
MicroscopyEducation
}

\section{A Leeuwenhoek-Type Mobile Microscope for Histology Education}

\author{
Masao Maeda, ${ }^{1,3 *}$ Nobuteru Usuda, ${ }^{1,3}$ Masahiro Kokubo, ${ }^{1}$ \\ Sumito Shirane, ${ }^{2}$ Motoaki Fukasawa, ${ }^{1}$ and Kuniaki Nagayama ${ }^{1,2,4, \dagger}$ \\ ${ }^{1}$ Department of Anatomy and Cell Biology, Fujita Health University School of Medicine, \\ Toyoake, Aichi, 470-1192, Japan \\ ${ }^{2}$ Science Communication Research Institute, Minami-ku, Yokohama, Kanagawa, 232-0006, Japan \\ ${ }^{3}$ Department of Biochemistry, Fujita Health University, Toyoake, Aichi, 470-1192, Japan \\ ${ }^{4}$ Life is Small Company, Minami-ku, Yokohama, Kanagawa, 232-0006, Japan
}

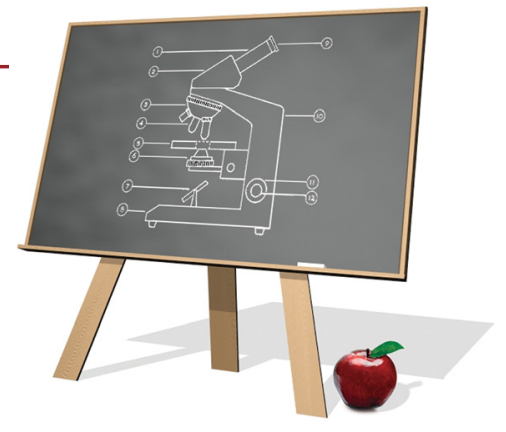

${ }^{\dagger}$ This author contributed equally to this work.

*masao.maeda@fujita-hu.ac.jp

\begin{abstract}
The smartphone microscope, or mobile microscope, is an interesting tool that is attracting attention in various fields. We report a model of mobile microscope that extends from the Leeuwenhoek-type of single microscope with a simple lens. The new mobile microscope is called the "L-eye mobile microscope" and is compact and easy to handle. The L-eye mobile microscope may become a hobby tool in everyday life for people of all ages but may also be a tool suitable in the education of medical or paramedical students. To test its potential as an educational tool, its performance was examined using samples such as permanent specimens of tissue sections from several organs used in histology and a standard target for a microscopy resolution test. As a control, we used a conventional education-use microscope that is mounted with a smartphone for image capture. From the performance comparison of the two kinds of microscope systems, the L-eye mobile microscope could take images with almost the same quality as those obtained with the conventional microscope at moderate magnifications. This result suggests the usefulness of the L-eye mobile microscope for education.
\end{abstract}

Keywords: mobile microscope, mobile device, single-lens microscope, histology, medical education

\section{Introduction}

Smartphone-based microscopes (also called mobile-phone microscopes) are in fashion for various purposes in several forms [1]. For example, the LudusScope is designed as an accessible, low-cost education kit and can be constructed and expanded with ease. It is a playful educational tool capable of observing the structure and dynamic behavior of live microorganisms [2]. Another simple, cost-effective smartphone microscope platform has been developed for secondary and high school students to obtain research-level images of parts of insects, pollen grains, and sodium chloride crystals [3]. One type of smartphone microscope is capable of identifying red and white blood cells in blood smears and soil-transmitted helminth eggs in stool samples [4], and another smartphone microscope has been used to detect malaria in fixed and stained blood smears $[5,6]$. The CellScope and Newton Nm1 smartphone microscopes can be used to detect protozoa for the diagnosis of intestinal diseases [7]. In the medical field, a tablet-based CellScope mobile microscope enables the remote diagnosis of oral cancer and may be used as a screening tool in pathology [8]. Thus, smartphone microscopes seem to be solidifying their ground in the medical and public health fields.
We developed a novel type of mobile microscope extended from the Leeuwenhoek (L) simple microscope with a combination of a single (simple or compound) lens and a smartphone (Figure 1). The new "L-eye mobile microscope" is compact and easy to handle, as it can be used while on a table and is free from blurring issues common with many types of mobile microscopes.

The aim of this study was to investigate whether the new microscope can complement the use of conventional compound microscopes, which have traditionally been used for histology education. We made a comparative study with two kinds of microscope systems: the L-eye mobile microscope and a conventional microscope used for histology. Both systems were specifically mounted with the same smartphone for image capture. Since our concern was the performance of the core part of each microscope, the same camera and mobile device were employed for image capture.

\section{Materials and Methods}

Animals and tissue preparation. For hematoxylin-eosin (H-E) staining, formalin-fixed skeletal muscle tissues of a postmortem male monkey (Macaca fuscata, five years old), which were kindly provided by Dr. Atsushi Nambu (National Institute for Physiological Sciences, NIPS), were washed with $0.1 \mathrm{M}$ sodium phosphate / $0.15 \mathrm{M}$ sodium chloride, $\mathrm{pH}$ 7.4, dehydrated in a graded ethanol and xylene series, and embedded in paraffin in a routine manner. Sections that were $4 \mu \mathrm{m}$ thick were made on a sliding microtome and collected on $0.7 \mathrm{~mm}$ thick glass slides. The sections were rehydrated with xylene and ethanol in series and stained with Mayer's hematoxylin and eosin Y (Merck, Darmstadt, Germany). For GolgiCox staining, a whole male adult Wistar rat brain (Japan SLC, Hamamatsu, Japan) was placed in Golgi-Cox solution [9] for two weeks and then sliced at $100 \mu \mathrm{m}$ thickness on a microslicer (Dohan EM, Osaka, Japan), and developed with ammonia solution. All sections were dehydrated in a graded ethanol and xylene series and mounted with Entellan New (Merck, Damstadt, Germany) with $0.17 \mu \mathrm{m}$ thick cover slips. The protocol for the monkey was carried out in compliance with the Animal Experimentation Regulations of the NIPS. The protocol 


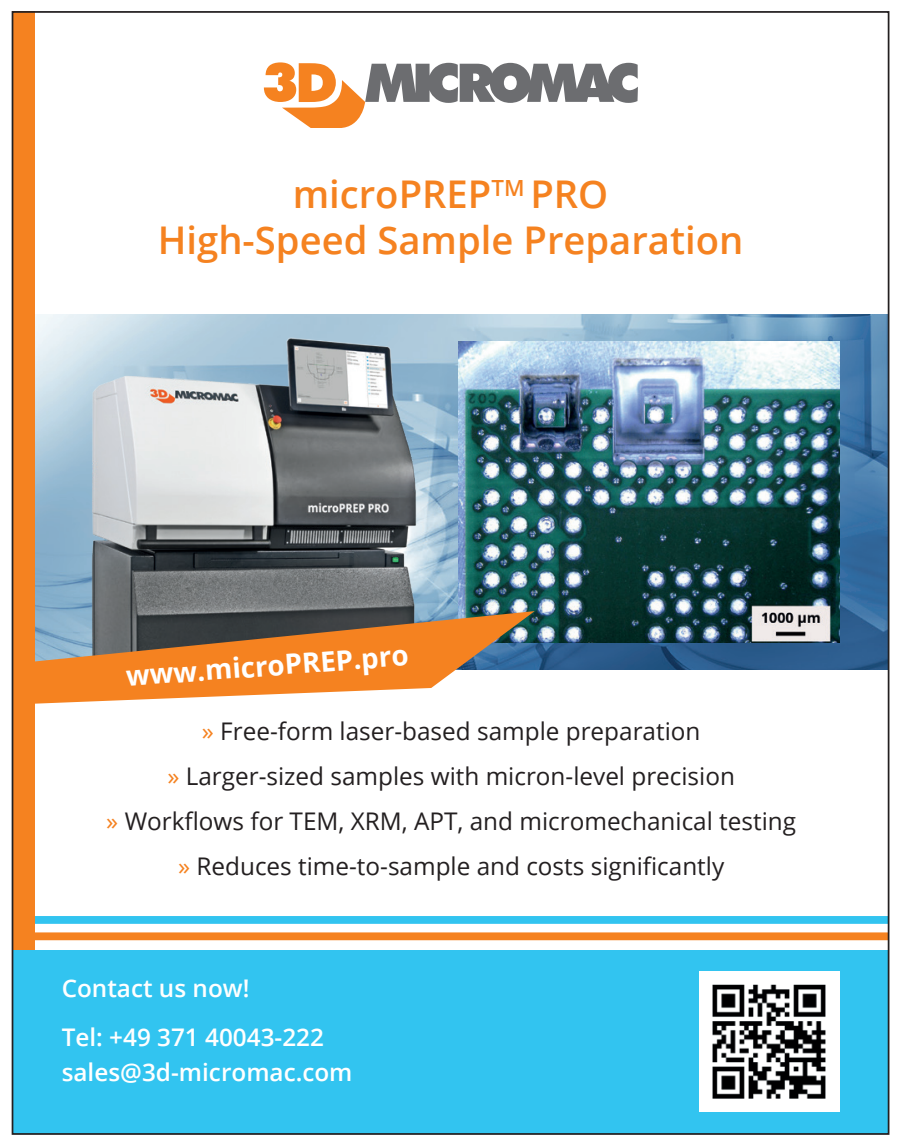

\section{TGS Technologies, LLC TEM Sample Holder Service and Modification Specialist}

With over 40 years of experience repairing TEM Sample Holders, we provide you with

high-quality personalized service. We specialize in repair, modification, and refurbishment of your sample holder as well as custom design if you are seeking specific innovative needs from your equipment.

TGS Technologies, LLC Ph: 724-453-3865 Fx: 724-453-2968

Email: tom@tgstechnologies.net http://www.tgstechnologies.net

\section{CAMBRIDGE}

New to the Advances in Microscopy and Microanalysis book series!

\section{Scanning Electron} Microscopy for the

\section{Life Sciences}

Heide Schatten

University of Missouri, Columbia

US\$120.00: Hb: 978-0-521-19599-7: $312 \mathrm{pp}$

Recent developments in scanning electron microscopy (SEM) have resulted in a wealth of new applications for cell and molecular biology, as well as related biological

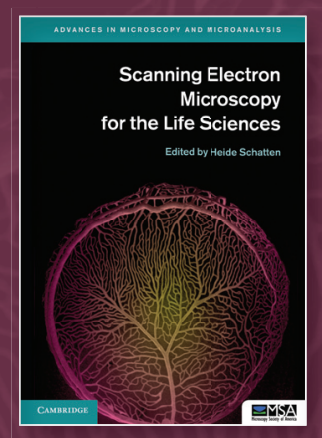
disciplines. It is now possible to analyze macromolecular complexes within their three-dimensional cellular microenvironment in near native states at high resolution, and to identify specific molecu les and their structural and molecular interactions. New approaches include cryo-SEM applications and environmental SEM (ESEM), staining techniques and processing applications combining embedding and resin-extraction for imaging with high resolution SEM, and advances in immuno-labeling. With chapters written by experts, this guide gives an overview of SEM and sample processing for SEM, and highlights several advances in cell and molecular biology that greatly benefited from using conventional, cryo, immuno, and high-resolution SEM.

\section{About the series}

The Press currently publishes the Microscopy and Microanalysis (MAM) journal in conjunction with the MSA, which reaches 4,000 microscopists and is affiliated with 12 international microscopy societies. The series would be a natural development from this journal, and will take a broad view of the discipline, covering topics from instrumentation to imaging, methodology and analysis across physical science, materials science, biology and medicine. Books commissioned for the series will range from advanced undergraduate textbooks through to research and practitioner oriented monographs for researchers. The series aims to produce a coherent source of material, encouraging the communication and exchange of ideas across these divergent fields, ensuring that the series appeals to a broad community in the physical and life sciences.

Forthcoming titles in this series:

Microscopic Nanocharacterization of Materials by Michael Isaacson

Energy Filtered Electron Microscopy and Electron Spectroscopy by Richard Leapman

Dynamic Transmission Electron Microscopy

by Nigel Browning, Thomas LaGrange, Bryan Reed,

Henning Stahlberg, Bradley Siwick 
(a)

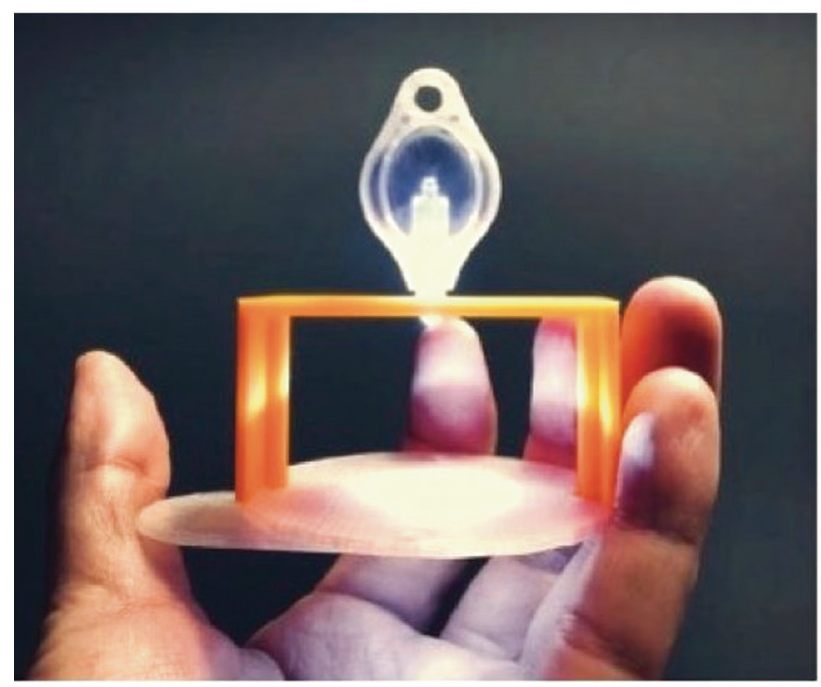

(c)

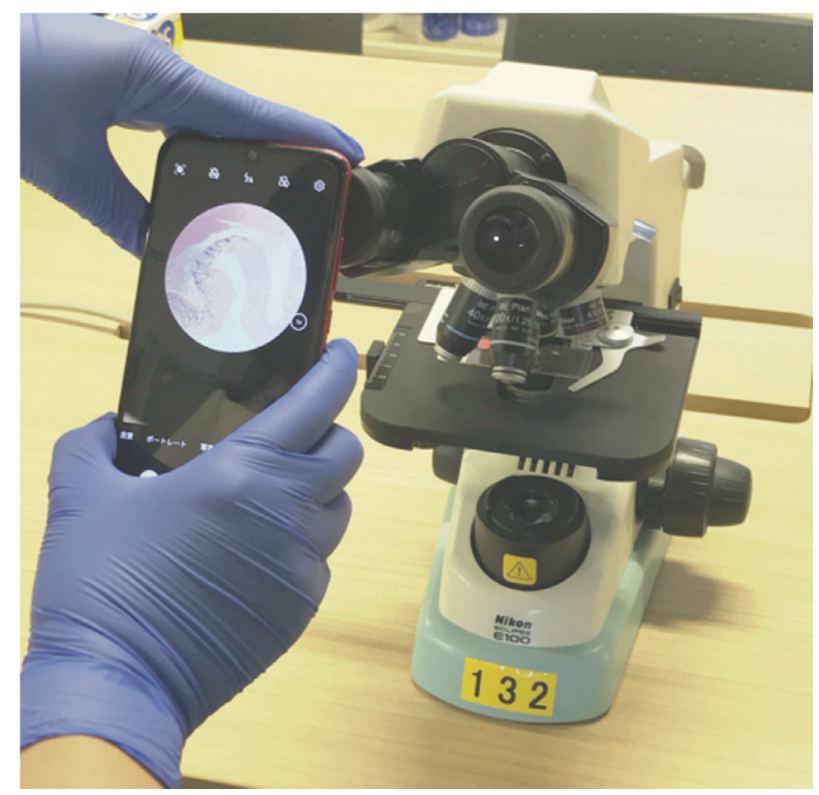

(b)

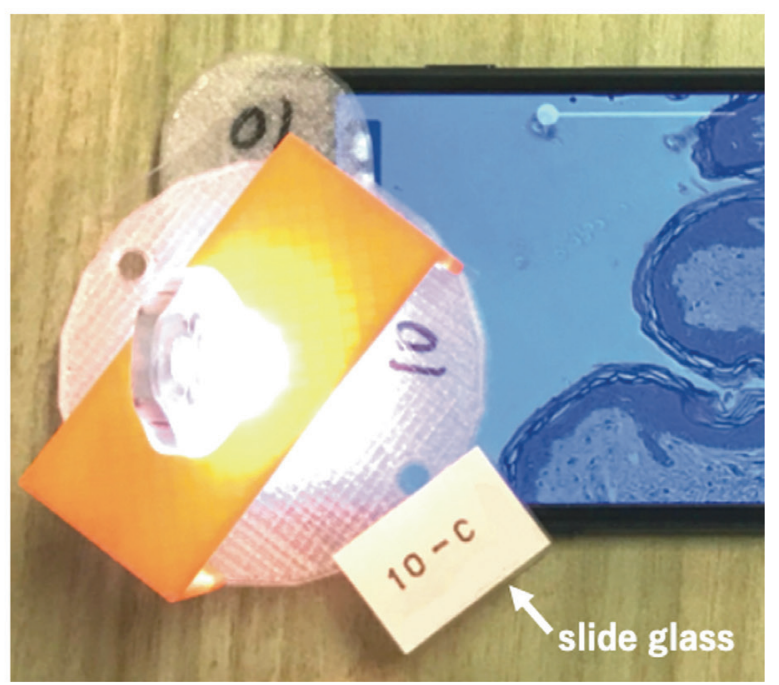

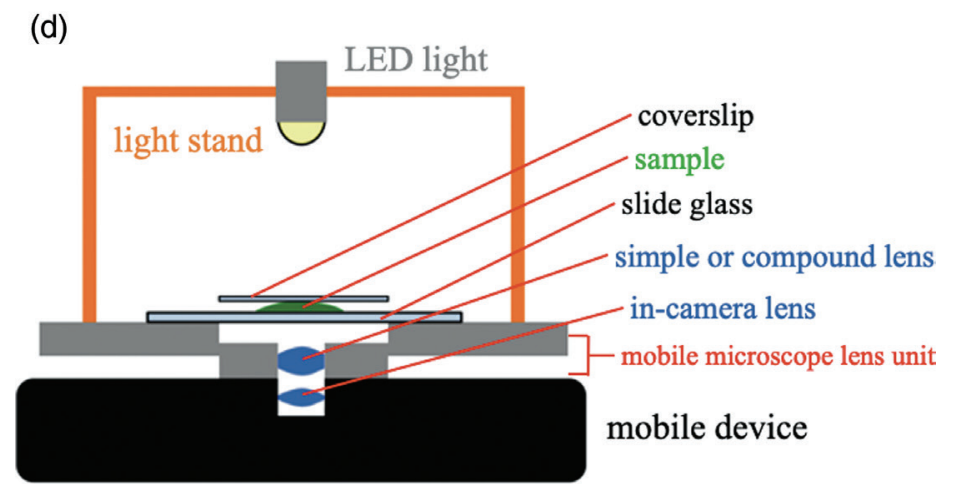

Figure 1: (a) An overview of the L-eye mobile microscope with an objective of $2.9 \mathrm{~mm}$ focal length. (b) A whole view of the L-eye microscope equipped with a smartphone (Huawei P10 Plus). (c) A conventional education-use microscope (ECLIPSE E100) that was mounted with a smartphone for image capture was the system used as a control. It was compared with the L-eye mobile microscope. (d) A schema of the side view of the L-eye microscope. The mobile microscope lens unit is a combination of a sample support made of a thin plastic disk with a female-screw in the center hole and a lens support holding a compound lens in the center with a male-screw outside. The screw mechanism enables focusing through the rotation of the thin-disk-attached light stand, which holds a LED.

for the rat was approved by the Institutional Animal Care and Use Committee (Protocol Number: \#AP16082) and carried out according to the Fujita Health University Animal Experimentation Regulations.

Microscopy observations. Two microscope systems were used for observation of the specimens. The first was a Leeuwenhoek-type mobile microscope (L-eye mobile microscope, Life is Small Company, Japan) (Figure 1), which includes a combination of an external lens made with a compound lens and a Huawei P10 Plus smartphone (Huawei P10 Plus, China). The other was a conventional microscope (ECLIPSE E100 microscope, Nikon, Japan) with the same Huawei P10 Plus smartphone directly attached to the eyepiece of the ECLIPSE E100. The mobile Huawei P10 Plus has three cameras: two back-facing cameras-one monochrome and one color-and a front-facing camera. While the color back-facing camera was mounted to the conventional microscope (ECLIPSE E100 microscope), the front-facing camera was used for the mobile microscope. The reason different cameras had to be used for the two microscope systems can be seen in Figures $1 \mathrm{~b}$ and $1 \mathrm{c}$, where the front-facing camera is an integrated part of the mobile microscope and the back-facing camera is that of the smartphone-mounted conventional microscope.

Specifications of the lenses tested are listed together with the other performance data in Table 1. Magnification of the 
Table 1: Specifications of lenses used for comparison of the L-eye mobile and ECLIPSE E100 microscopes.

\begin{tabular}{|l|c|c|c|c|c|}
\hline & $\begin{array}{c}\text { Focal length } \\
(\mathbf{m m})\end{array}$ & Magnification & $\begin{array}{c}\text { NA } \\
\text { (numerical aperture) }\end{array}$ & $\begin{array}{c}\text { Theoretical } \\
\text { resolution }\end{array}$ & $\begin{array}{c}\text { Working } \\
(\mu \mathrm{m})\end{array}$ \\
distance $(\mathbf{m m})$
\end{tabular}

a: $0.61 \lambda / \mathrm{NA}(\lambda=589.6 \mathrm{~nm}$ ( $\mathrm{NaD}$ line)).

b: Maker's specification for the objective lens.

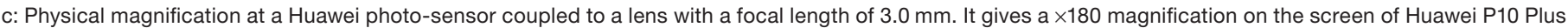
with $\times 6$ pinch-out.

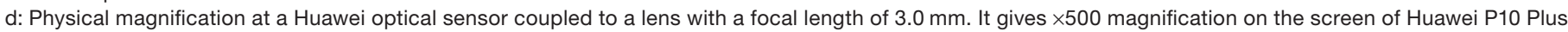
with $\times 6$ pinch-out.

e: A lens supplied from a Japanese company that is manufacturing small compound lenses for mobile cameras.

L-eye mobile microscope was adjusted by changing the focal length as shown in Table 1, and magnifications of $\times 180$ and $\times 500$ were used to test resolution. For the ECLIPSE E100 microscope, $10 \times$ and $40 \times$ objectives with a $10 \times$ intermediate lens were used for final magnifications of $\times 100$ and $\times 400$. For the resolution comparison for the two microscopes, the Edmund Optics (Barrington, NJ) test target was employed (Figure 2), and for the image quality investigation, tissue sections were imaged (Figures 3 and 4).
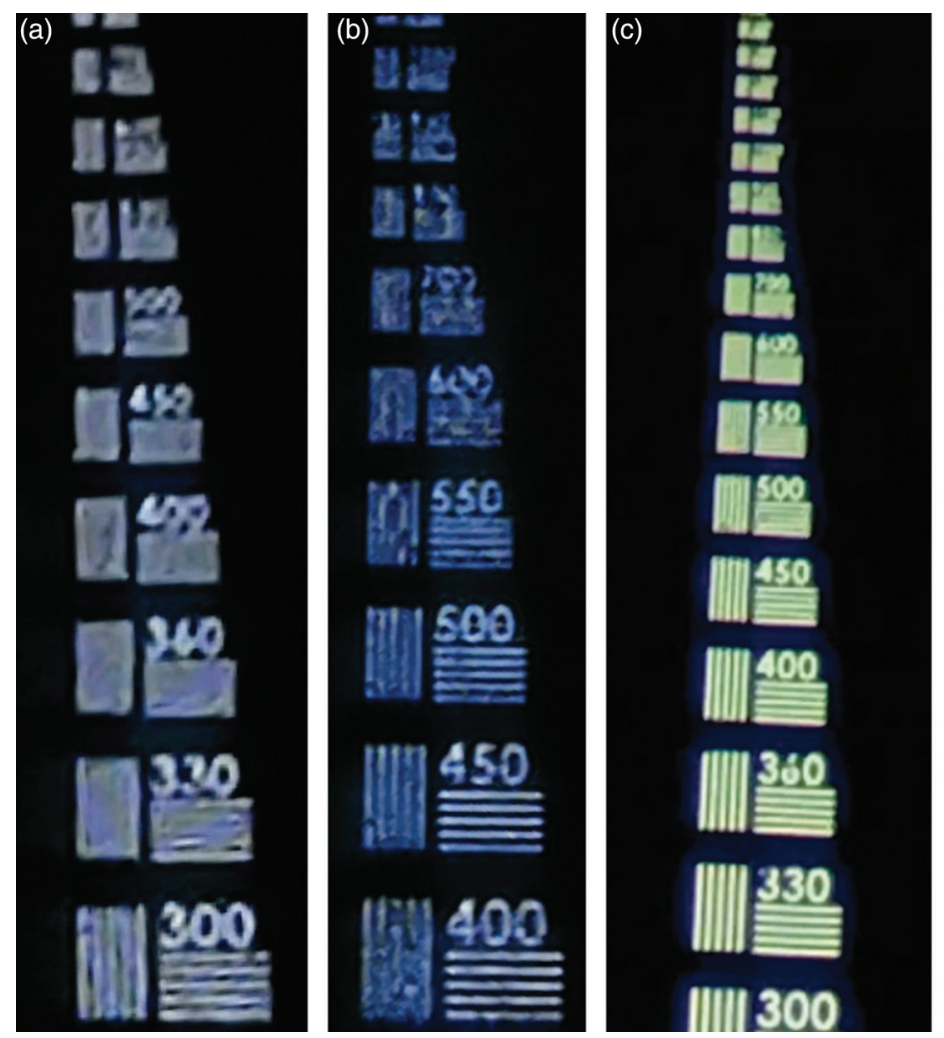

Figure 2: Images of a microscopy test target (Edmund) taken with (a) L-eye mobile microscope with an objective of $2.9 \mathrm{~mm}$ focal length ( $\times 180$ magnification on the screen) has a resolution of $500 \mathrm{lp} / \mathrm{mm}$ or $2.0 \mu \mathrm{m}$; (b) L-eye mobile microscope with an objective of $0.75 \mathrm{~mm}$ focal length ( $\times 500$ magnification on the screen) has a resolution of $700 \mathrm{lp} / \mathrm{mm}$ or $1.4 \mu \mathrm{m}$; (c) the ECLIPSE E100 with a $10 \times$ objective lens ( $\times 100$ magnification) has a resolution of $600 \mathrm{lp} / \mathrm{mm}$ or $1.8 \mu \mathrm{m}$, and (d) the ECLIPSE E100 with a $40 \times$ objective lens ( $\times 400$ magnification) has a resolution of $1100 \mathrm{lp} / \mathrm{mm}$ or $0.9 \mu \mathrm{m}$. Resolution with the L-eye mobile microscope is not quite as good as with the ECLIPSE E100 but sufficient for initial education in histology. Both microscopes were mounted with the same smartphone device (Huawei P10 Plus).

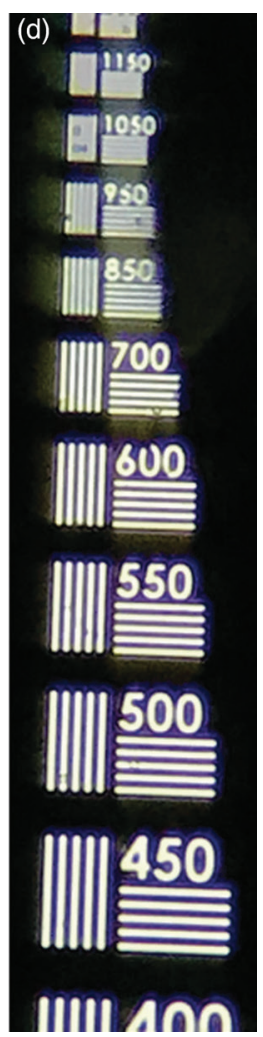

\section{Results}

Prior to the experiments, we tested the three cameras in the Huawei smartphone for image acquisition and found no obvious difference in image quality among them (data not shown).

The performance test with the Edmund test target is illustrated in Figure 2. The resolution of the $\times 180(2.9 \mathrm{~mm}$ focal length) mobile microscope is approximately $500 \mathrm{lp} /$ $\mathrm{mm}(2.0 \mu \mathrm{m}$ resolution $)$, and that of the $\times 500$ mobile microscope $(0.75 \mathrm{~mm}$ focal length) is approximately $700 \mathrm{lp} / \mathrm{mm}(1.4 \mu \mathrm{m}$ resolution). Resolution of the $\times 100$ ECLIPSE microscope (with a $10 \times$ objective lens) is about $600 \mathrm{lp} / \mathrm{mm}$ (1.8 $\mu \mathrm{m}$ resolution), and that of the $\times 400$ ECLIPSE microscope (with a $40 \times$ objective lens) is about 1100 $\mathrm{lp} / \mathrm{mm}(0.9 \mu \mathrm{m}$ resolution). The resolution of the $\times 180 \mathrm{~L}$-eye microscope is comparable to that of the $\times 500$ L-eye microscope and slightly better than the $\times 100$ ECLIPSE microscope, but the resolution of the $\times 500 \mathrm{~L}$-eye microscope is worse than that of the $\times 400$ ECLIPSE microscope.

Two types of sections customarily used for histology in medical education at Fujita Health University were embedded between a glass slide and a cover glass and examined with the L-eye $(\times 180$ and $\times 500)$ and educational $(10 \times$ objective lens and $40 \times$ objective lens) microscopes. Figure 3 shows skeletal muscle from a monkey where characteristic long muscle fibers are seen with both the L-eye and educational microscopes. The striations of skeletal muscle fibers are visible with the $\times 500 \mathrm{~L}$-eye microscope but not resolved with the $180 \times$ L-eye microscope (Figure 3a), which are compared with results obtained with the educational microscope (Figure 3b), where they are visible with the $40 \times$ objective lens but not resolved with the $10 \times$ lens. Figure 4 shows tissue from rat cerebrum where 
(a)

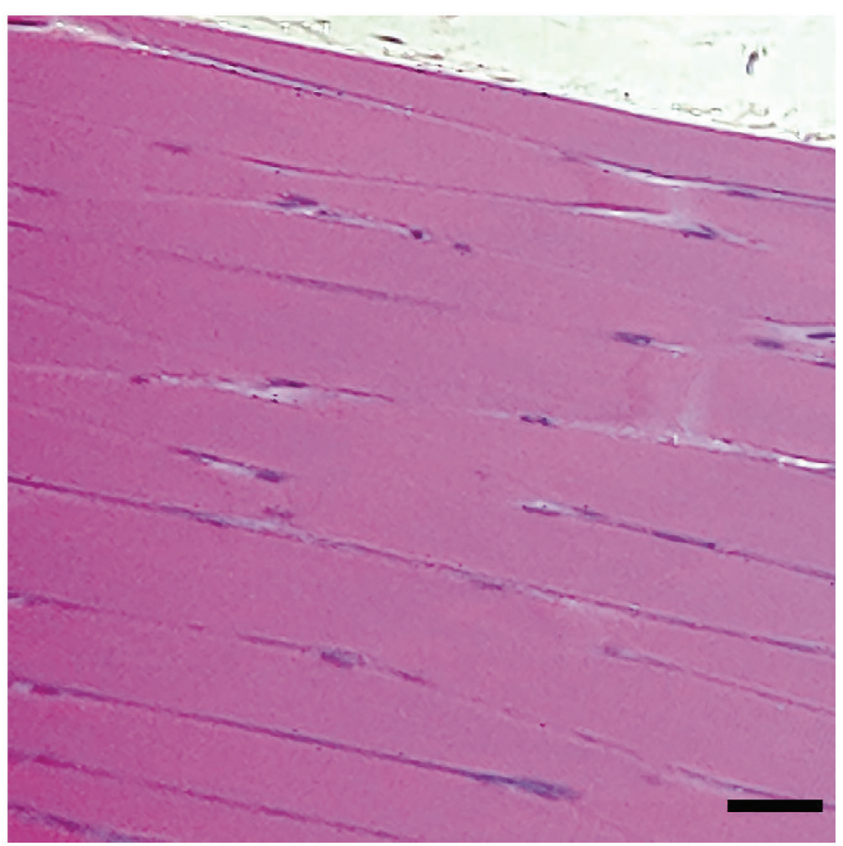

(c)

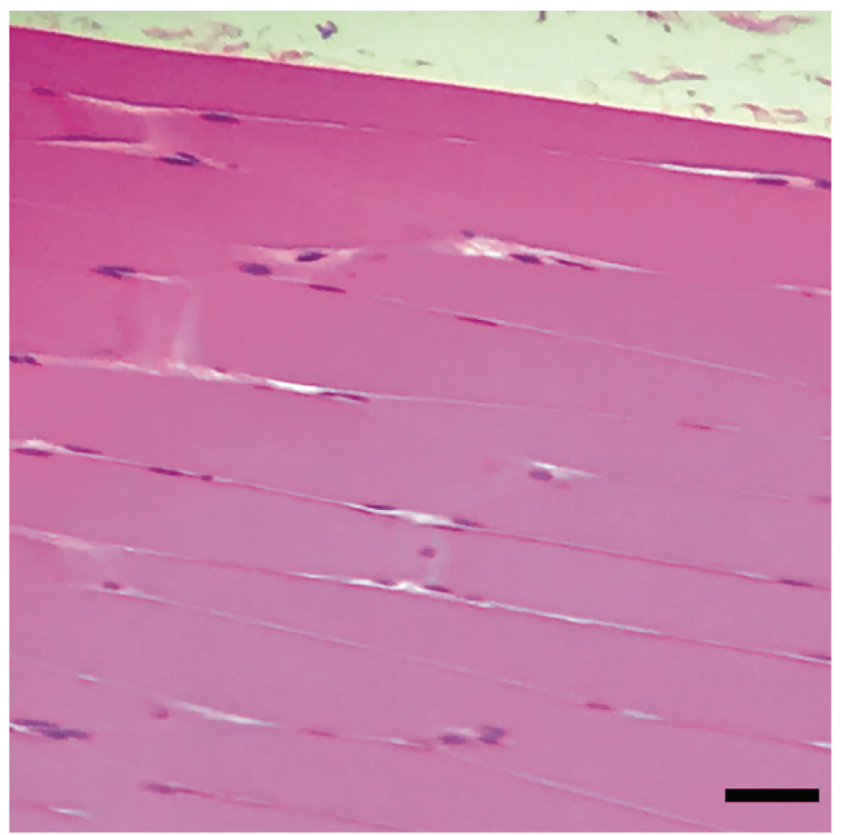

(b)

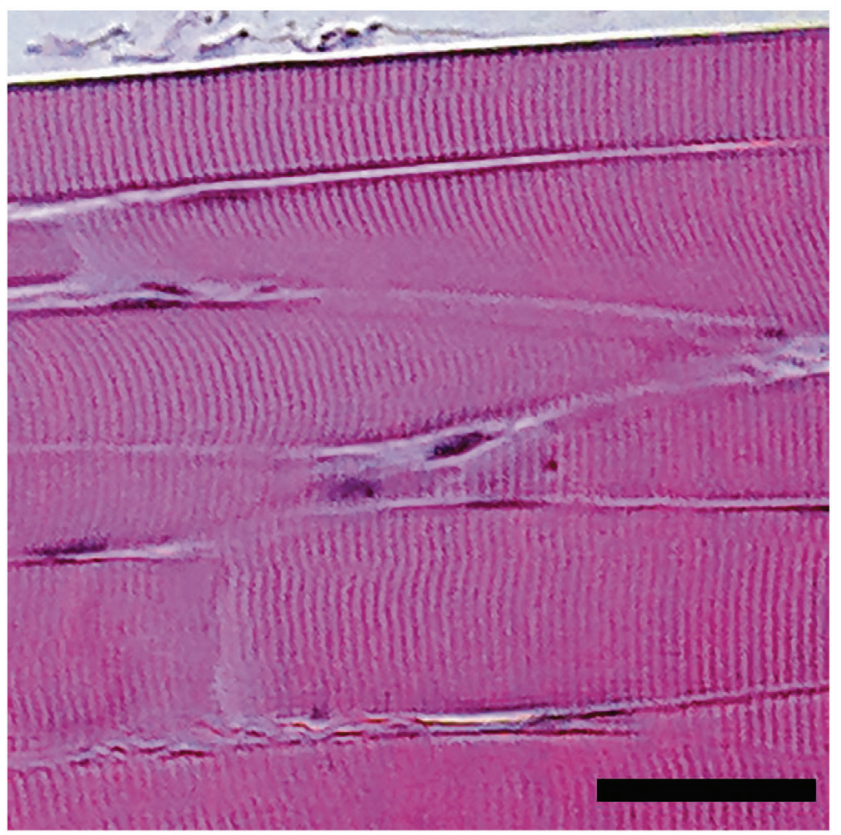

(d)

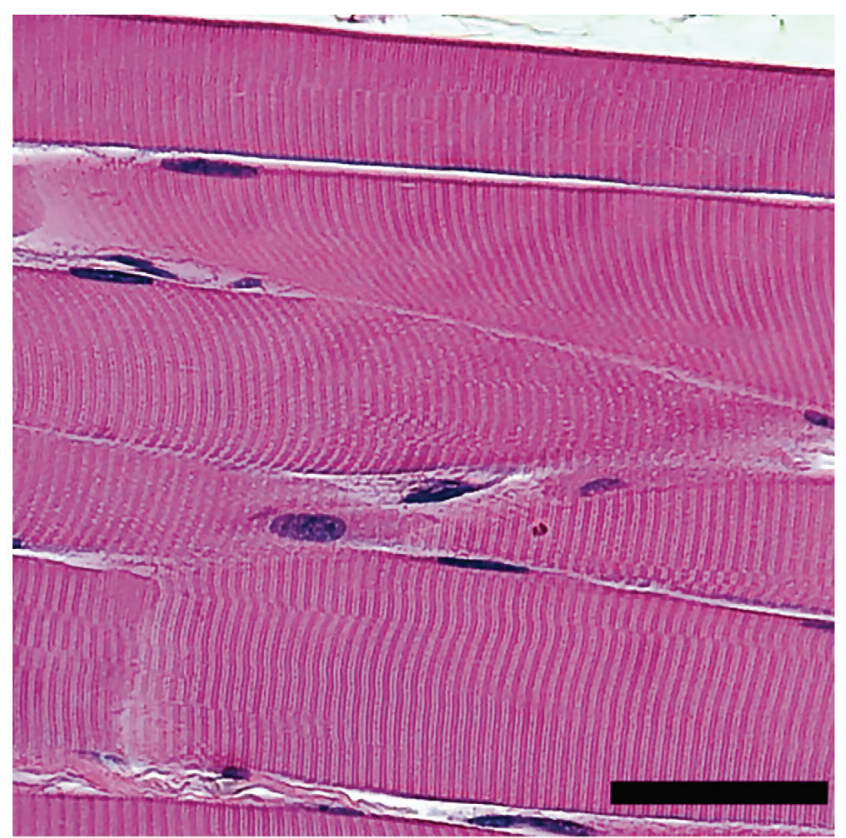

Figure 3: Images of $4 \mu \mathrm{m}$ thick sections of monkey skeletal muscle with hematoxylin-eosin staining. (a), (b) An image taken with the L-eye mobile microscope, $\times 180$ (a) and $\times 500$ (b). At the lower magnification muscle striations are not resolved, but with the $\times 500$ magnification striations are visible. (c), (d) Images taken with the educational ECLIPSE E100 microscope with 10× (c) and 40× (d) objective lenses. As with the L-eye mobile system, striations are not visible at the lower resolution. All images were collected with the Huawei P10 Plus smartphone. Scale bars represent $50 \mu \mathrm{m}$.

pyramidal cells with dendrites from the cell bodies are visible as a meshwork with both the L-eye mobile and ECLIPSE E100 microscopes. With the $\times 180 \mathrm{~L}$-eye microscope all neurons in this thick section could be focused in the same image (Figure 4a). However, in the image from the ECLIPSE E100 microscope with a $10 \times$ objective lens, some neurons are focused, but others are out of focus (Figure $4 \mathrm{~b}$ ). The neurons imaged in this $100 \mu \mathrm{m}$ section of brain are located at different distances from the objective.

\section{Discussion}

The resolution and image quality obtained by the L-eye mobile microscope are comparable to those obtained with the ECLIPSE E100. The resolution of the $\times 500$ mobile microscope is sufficient to observe skeletal muscle fiber striations, which are not resolved with an educational microscope with a $10 \times$ objective (Figure 3). The quality of the mobile microscope images for histological sections is good enough for initial histology education and understanding tissue structure. Furthermore, with 
(a)

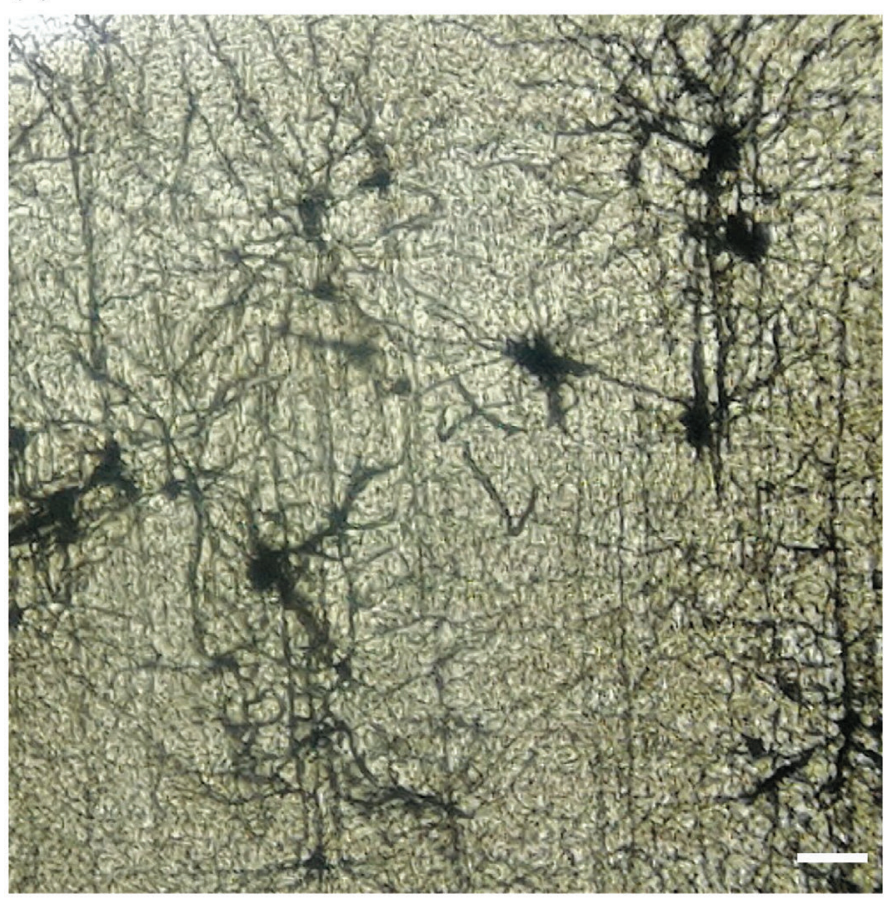

(b)

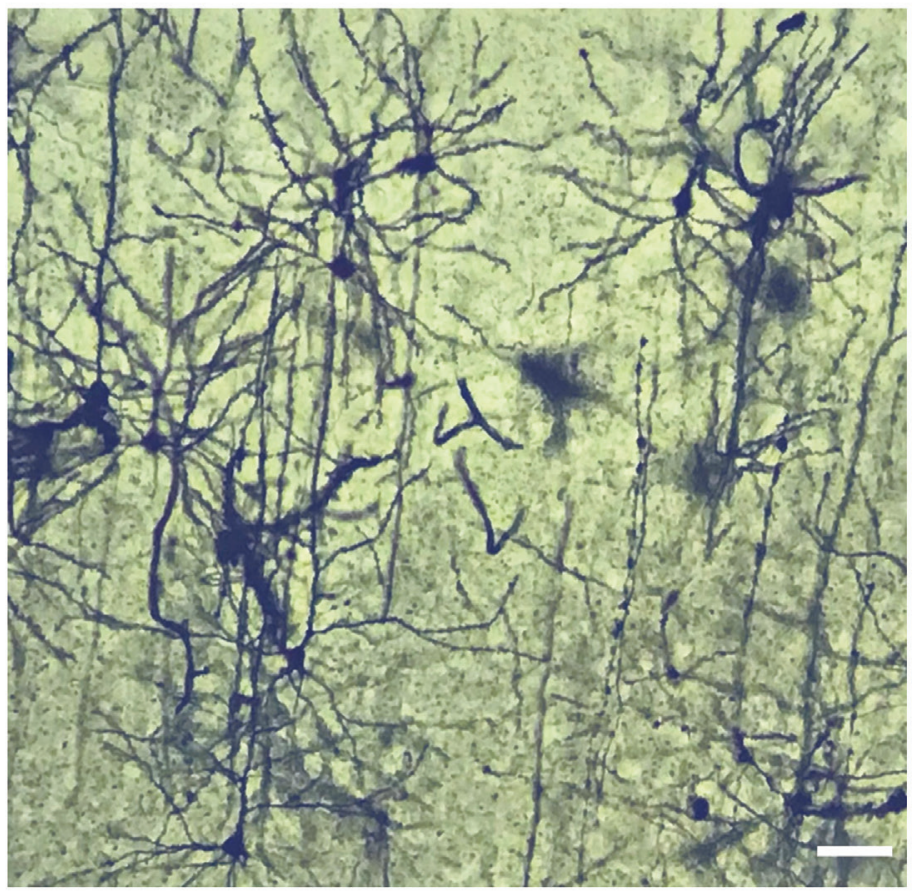

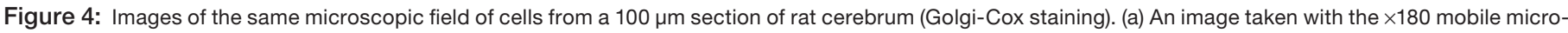

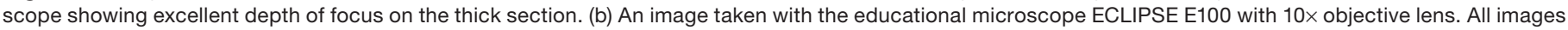
were collected with the Huawei P10 Plus smartphone. Scale bars represent $50 \mu \mathrm{m}$.

the educational microscope with a $10 \times$ objective lens, all neurons are not necessarily focused in a single image (Figure 4b), but with the $\times 180 \mathrm{~L}$-eye microscope they are focused in a single image (Figure $4 \mathrm{~b}$ ). The deeply focused image presentation of histological sections may make it easier for students to understand tissue structure.

Performance of the L-eye mobile microscope configured as a Leeuwenhoek-type system with the original small size was comparable to a conventional light microscope for histological observation. In contrast, most other smartphone microscopes are based on the combination of mobile devices and a traditional compound microscope, where the smartphone camera attaches to a long tube that holds intermediate and objective lenses and has the problem of blurring when taking a picture by hand. The blurring can be fixed with the use of a special bulky console, but only by sacrificing the original compactness. Compared to those smartphone microscopes, the L-eye mobile microscope may be distinguished by several characteristics: it is compact, light, portable, easy to operate and maintain, and, most importantly, free from the blurring issue as it is set statically on a table.

Many medical schools utilize one microscope for each student in histology and pathology classes. However, at paramedical educational sites such as nursing schools, a sufficient number of ordinary microscopes may not be available. Since the performance of the L-eye mobile microscope at a moderate magnification was found to be high enough to be practically employed, it could provide a handy and cost-effective substitute for standard microscopes used in nursing and paramedical settings. Furthermore, histology education with the L-eye mobile microscope can be extended to primary and secondary schools, to lectures for lay audiences, and to general use in underdeveloped countries where expensive microscopes are difficult to obtain.

\section{Conclusions}

The image quality, resolution, and colors of the pictures taken by the L-eye microscope, in spite of its simple construction, were comparable to those obtained by the conventional microscope used in medical education. The L-eye mobile microscope is convenient to use in medical, nursing, and paramedical educational settings as a secondary device, where the necessary number of ordinary microscopes are not available.

\section{Acknowledgments}

The authors are grateful to Prof. Atsushi Nambu of the National Institute for Physiological Science for providing tissue sections. We also would like to thank Mr. Toshiyuki Itoh for his continuous support for manufacturing mobile microscopes.

\section{References}

[1] JM Perkel, Nature 545 (2017) 119-21.

[2] H Kim et al., PLoS One 11 (2016) e0162602.

[3] LC Wicks et al., Wellcome Open Res 2 (2018) 107.

[4] A Skandarajah et al., PLoS One 9 (2014) e96906.

[5] CW Pirnstill and GL Cote, Sci Rep 5 (2015) 13368.

[6] TE Agbana et al., PLoS One 13 (2018) e0205020.

[7] JT Coulibaly et al., PLoS Negl Trop Dis 10 (2016) e0004768

[8] A Skandarajah et al., PLoS One 12 (2017) e0188440.

[9] Y Koyama et al., J Neurosci Methods 218 (2013) 103-09. 